\title{
De-intensification strategies in HPV-related oropharyngeal squamous cell carcinoma-a narrative review
}

\author{
Madeleine P. Strohl, Katherine C. Wai, Patrick K. Ha \\ Division of Head and Neck Surgical Oncology, Department of Otolaryngology-Head and Neck Surgery, University of California, San Francisco, \\ CA, USA \\ Contributions: (I) Conception and design: All authors; (II) Administrative support: PK Ha; (III) Provision of study materials or patients: PK Ha; \\ (IV) Collection and assembly of data: MP Strohl, KC Wai; (V) Data analysis and interpretation: MP Strohl, KC Wai; (VI) Manuscript writing: All \\ authors; (VII) Final approval of manuscript: All authors. \\ Correspondence to: Patrick K. Ha, MD. Division of Head and Neck Surgical Oncology, Department of Otolaryngology-Head and Neck Surgery, \\ University of California, 550 16th St, 6th Floor, San Francisco, CA 94158, USA. Email: Patrick.ha@ucsf.edu.
}

\begin{abstract}
Human papillomavirus-related (HPV+) oropharyngeal squamous cell carcinoma (OPSCC) is a relatively new clinical entity that is dramatically on the rise globally. HPV+ OPSCC is thought to be a separate clinical entity compared to HPV- OPSCC with a distinct tumor biology. Patients with HPV associated disease have been shown to have a substantially better prognosis and overall survival than those patients with the HPV negative (HPV-) counterpart. The standard of care for OPSCC is definitive radiation therapy (RT) and concurrent chemoradiation therapy (CRT), for lower and higher stage disease, respectively. However, traditional CRT is also associated with severe acute and late toxicities affecting patient quality of life, such as severe mucositis, dry mouth and dysphagia. Considering that HPV+ OPSCC is on the rise in a younger, healthier patient population and the good prognosis of HPV-related disease, there has been a focus on reducing treatment toxicities and optimizing quality of life while maintaining favorable oncologic outcomes. A variety of such de-escalation regimens are currently being explored in recently completed and ongoing clinical trials. Alterations to the standard chemotherapy, radiation and surgical regimens are being explored. This review will provide an overview of the rationale for and available results of the major deintensification strategies in the treatment of locally advanced HPV+ OPSCC.
\end{abstract}

Keywords: Human papilloma virus; oropharyngeal cancer; de-intensification

Submitted Mar 30, 2020. Accepted for publication Sep 25, 2020.

doi: $10.21037 / \mathrm{atm}-20-2984$

View this article at: http://dx.doi.org/10.21037/atm-20-2984

\section{Introduction}

Human papillomavirus-related (HPV+) oropharyngeal squamous cell carcinoma (OPSCC) is a relatively new clinical entity that is dramatically on the rise globally (1). $\mathrm{HPV}+$ OPSCC is thought to be a separate clinical entity compared to HPV- OPSCC with a distinct tumor biology. About $60 \%$ of oropharynx cancers are associated with HPV infection. The incidence has been increasing in recent years, especially in individuals under the age of 45 . This change is attributed to the increasing prevalence of HPV infection in developed countries, the practice of oral sex, and the rising number of sexual partners. HPV+ OPSCC is mediated by infection with the human papilloma virus, which targets the p53 and pRb tumor suppressor pathways and results in cell cycle alteration.

Patients with both HPV+ and HPV- disease can present with vague symptoms including sore throat, dysphagia, odynophagia or ear pain. However, patients presenting with $\mathrm{HPV}+$ disease are usually younger, healthier and without the usual risk factors of aerodigestive tract malignancy such as significant smoking or alcohol use. Additionally, patients with HPV+ disease are often relatively asymptomatic, presenting instead with a painless neck mass and no other 

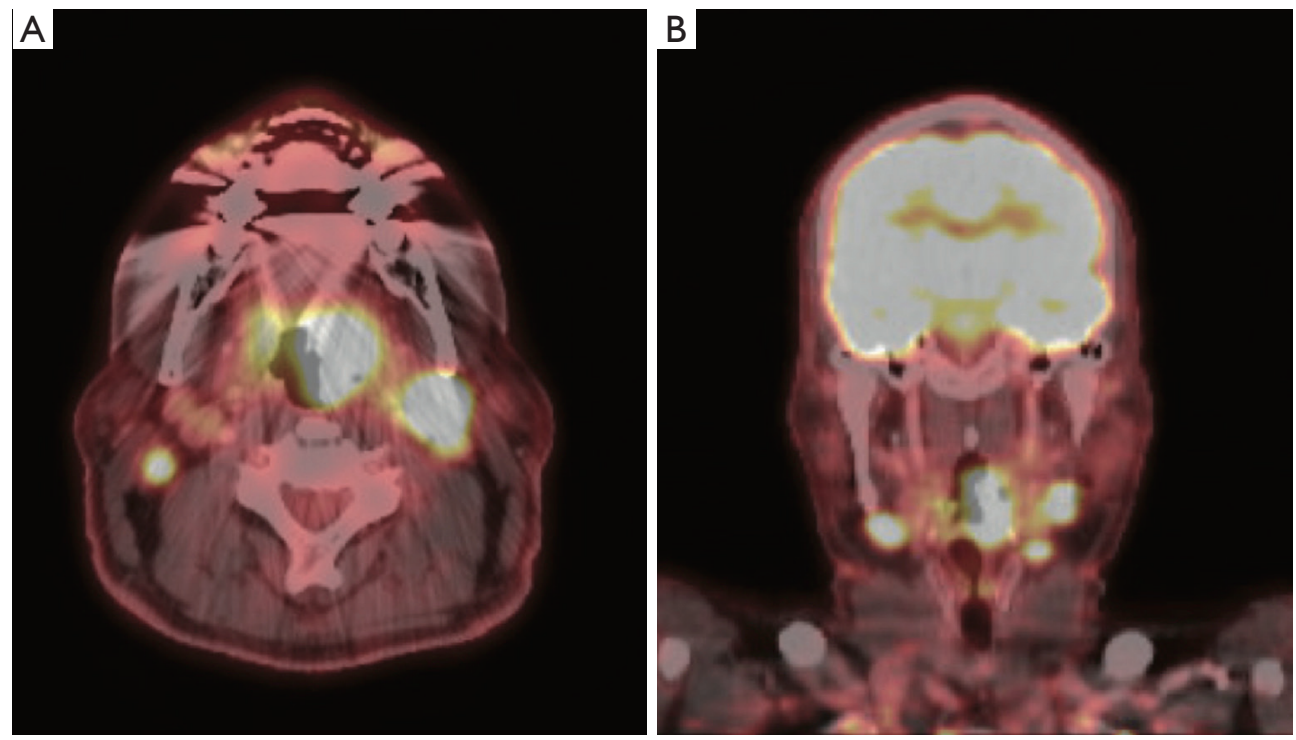

Figure 1 Positive emission tomography/computer tomography (PET/CT) of a patient with locally advanced base of tongue HPV+ OPSCC. (A) Axial view. (B) Coronal view.

symptoms. Fortunately, patients with HPV+ OPSCC have been shown to have a substantially better prognosis and overall survival than those with the HPV- counterpart, even when presenting with similar disease burden (2). A separate TNM staging system through the American Joint Committee on Cancer (AJCC) $8^{\text {th }}$ edition has been developed resulting in downstaging of many patients with $\mathrm{HPV}+$ OPSCC. Figure 1 shows a representative positive emission tomography/computer tomography (PET/CT) of a patient with locally advanced base of tongue HPV+ OPSCC. This patient has a left sided base of tongue tumor and bilateral enlarged, hypermetabolic cervical lymph nodes. By the AJCC $8^{\text {th }}$ edition, this patient would be stage II.

While there are different staging systems for HPV+ and HPV- disease, current treatment strategies are the same for both entities. The standard of care for both subtypes of OPSCC is definitive radiation therapy (RT) and concurrent chemoradiation therapy (CRT), for lower and higher stage disease, respectively. However, traditional RT and CRT are also associated with severe acute and late toxicities affecting patient quality of life, such as severe mucositis, dry mouth and dysphagia.

De-intensification, or the reduction in some element of the cancer treatment regimen, is of great interest in HPV+ OPSCC. Considering that HPV+ OPSCC commonly presents in a younger, healthier patient population compared to HPV-negative and the good prognosis of
HPV related disease, there has been a focus on reducing treatment toxicities and optimizing quality of life while maintaining favorable oncologic outcomes. As a result, several de-intensification regimens have been proposed in select patient populations. In the past several years alone, there have been numerous studies and trials investigating de-intensification therapy. The recent completion of clinical trials such as the RTOG-1016 trial, the De-SCALaTE HPV trial, and the ORATOR trial have produced interesting and exciting results.

A variety of such de-escalation regimens are currently being explored in recently completed and ongoing clinical trials (Table 1). This review provides a modern overview of the rationale for and available results of the major de-intensification strategies in the treatment of locally advanced HPV+ OPSCC. Great advances have been made in the past five years, and recent completion of a number of large clinical trials on de-escalation in the. past couple years have improved our understanding of the treatment of this disease. We present the following study in accordance with the Narrative Review reporting checklist (available at http:// dx.doi.org/10.21037/atm-20-2984).

\section{Replacement of cisplatin-based chemotherapy with cetuximab}

Several clinical trials have evaluated the potential of the 


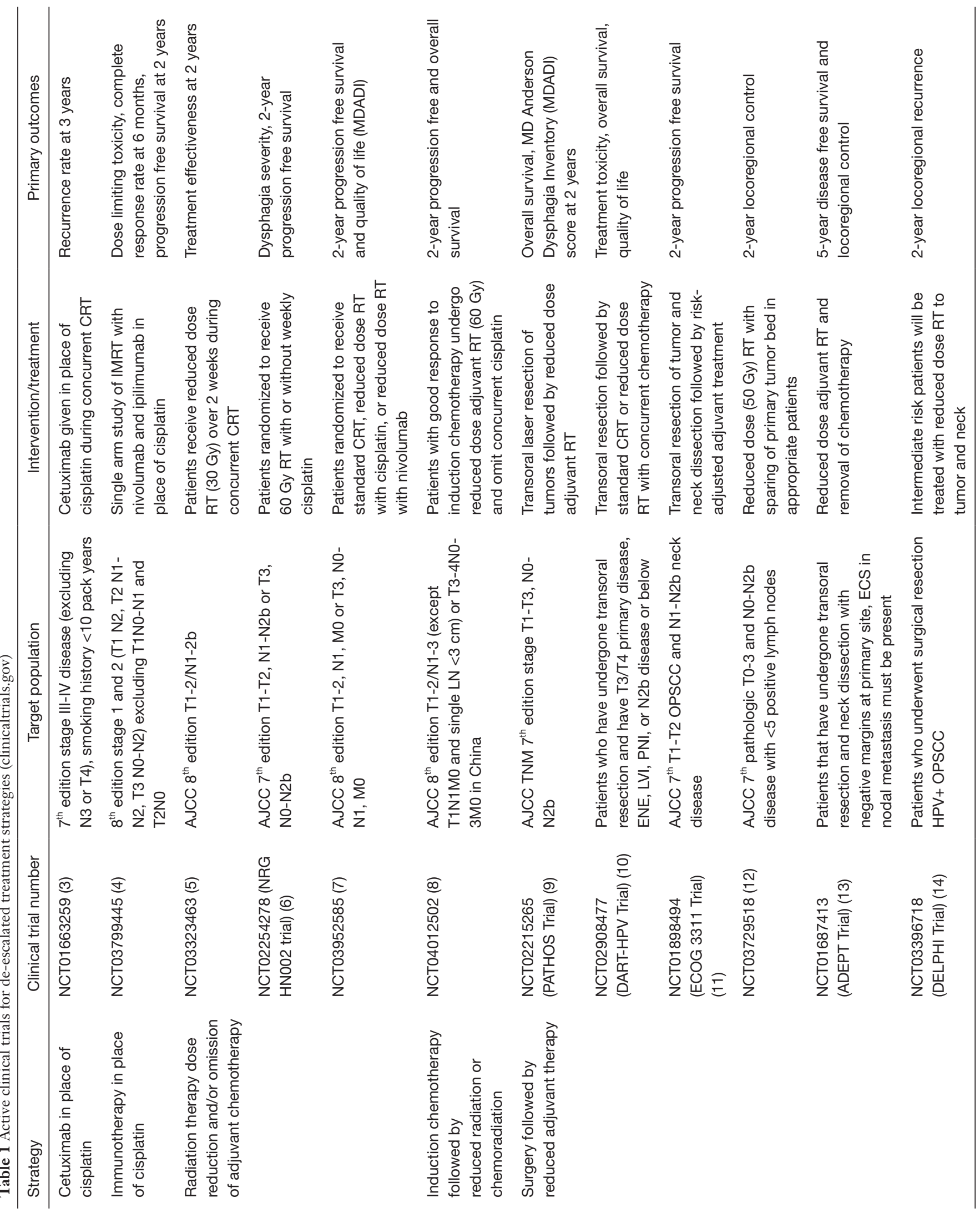


monoclonal anti-EGFR antibody known as cetuximab in place of cisplatin during CRT. The rationale for cetuximab as a treatment for OPSCC was based on Bonner et al.'s 2006 article which reported improved survival rates after addition of cetuximab to RT compared to RT alone for patients with upper aerodigestive squamous cell carcinoma in the IMBL9815 trial (15). While this study did not specifically look at OPSCC or HPV status, a subgroup analysis in 2010 showed that the benefit conferred through cetuximab was most evident in OPSCC, in younger patients and in those with a better Karnofsky performance score, which pointed towards effectiveness in HPV+ tumors (16). It was thought that cetuximab is less toxic than cisplatin-based chemotherapy; although no study at the time compared cetuximab directly with cisplatin.

The pre-clinical studies of EGFR inhibition in oropharyngeal carcinoma are largely analyzing the role of EGFR-inhibition in HPV-negative disease rather than $\mathrm{HPV}$-positive disease. Currently, there is no preclinical data showing a radiosensitizing effect of cetuximab when added to radiation for HPV-related oropharyngeal cancer. The rationale for EGFR-inhibition in HPV-related oropharyngeal carcinoma is controversial, as it is thought that HPV-related tumors are less driven by such alterations in cell signaling pathways due to the oncogenic properties of HPV-oncoproteins E6 and E7. When compared to $\mathrm{HPV}$-negative carcinoma, HPV-related OPSCC has been shown to harbor fewer driver mutations or alterations such as EGFR-overexpression (17).

Two phase III clinical trials studying the effectiveness of cetuximab in place of cisplatin during concomitant radiation for patients with HPV+ OPSCC have recently published results reporting inferiority of this approach. In the RTOG1016 study, Gillison et al. [2019] randomized 849 patients to receive cetuximab or cisplatin during chemoradiation. The cetuximab group had a significantly inferior five-year overall survival compared to the cisplatin group $(77.9 \%$ versus $84.6 \%, \mathrm{P}=0.0163$, HR 1.45). Interestingly, there was no significant difference between the rates of acute moderate to severe toxicity $(77.4 \%$ versus $81.7 \%, \mathrm{P}=0.1586)$ and late moderate to severe toxicity ( $16.5 \%$ versus $20.4 \%, \mathrm{P}=0.1904$ ) for the cetuximab and cisplatin groups, respectively. This was surprising given that this trial was based on the idea that cetuximab would provide a reduction in toxicity compared to cisplatin, with comparable survival (18).

In the De-SCALaTE HPV trial, Mehanna et al. [2019] randomized 334 patients with locally advanced HPV+ OPSCC with a less than 10 pack year smoking history to receive a chemoradiation regimen with cisplatin or cetuximab. Similar to the RTOG-1016, treatment arms did not differ in the rate of severe and overall acute or late toxicities. However, two-year overall survival was significantly decreased for cetuximab compared to cisplatin ( $89.4 \%$ versus $97.5 \%$, respectively, with $\mathrm{P}=0.00012$ and HR 5) and two-year recurrence rate was also significantly increased for cetuximab (16.1\% for cetuximab versus 6\% for cisplatin, $\mathrm{P}=0.0007$, HR 3.4). Two-year overall survival rates were statistically significantly different even among patients with stage I/II disease (19).

A third clinical trial (NCT01663259) evaluating cetuximab in place of cisplatin is enrolling patients and has not yet reported results. The trial began in 2011 and recently completed enrolling patients in September 2019. In this study at the University of Michigan, patients with stage III and IV (excluding N3 and T4) HPV related OPSCC and smoking history $<10$ pack years will receive induction cetuximab followed by concurrent cetuximab and RT at 70 Gy (3). Further trials examining cisplatinineligible patients are more important given this finding, as we know that cetuximab does not impart a survival benefit in this population.

\section{Immune checkpoint inhibitors in place of cisplatin-based chemotherapy}

Another avenue has been to evaluate the use of immune checkpoint inhibitors in place of conventional chemotherapy. Patients with HPV-related OPSCC are thought to be ideal candidates for immunotherapy as oncogenesis is driven by blockade of the innate immune system. The programmed cell death pathway has been shown to play a role in persistent HPV infection and resistance to elimination by the immune system during malignant progression of disease. Increased levels of programmed death ligand-1 have been shown in HPV-related OPSCC and have also been significantly associated with survival (20). Immunotherapy with monoclonal antibodies targeting these receptors, including immune checkpoint inhibitors ipilimumab and nivolumab, have become standard of care in recurrent or metastatic head and neck squamous cell carcinoma after platinum-based immunotherapy. Additionally, the side effect profile is different and thought to be reduced compared to standard chemotherapy. Common side effects include diarrhea, skin rash, itchiness, pneumonitis, kidney infections and changes in hormone levels. A phase II clinical study with the National Cancer Institute and University of 
Texas MD Anderson Cancer Center is ongoing to test the safety, tolerability and efficacy of ipilimumab and nivolumab in combination with radiation (up to $60 \mathrm{~Gy}$ ) in patients with early stage HPV related OPSCC, including patients with T1N2, T2N1-2 and T3N0-2 disease (4). Again, due to the inferiority of cetuximab, the potential use of checkpoint inhibitors is appealing to maximize survival and minimize side effects. Immune modulation represents an exciting new line of therapy that will be further investigated for years to come.

\section{$\mathbf{R T}$ dose reduction and/or omission of adjuvant chemotherapy}

There is a well-established correlation between cumulative RT dose delivered and short and long-term morbidity. Traditional side effects of RT to the head and neck include dry mouth, mouth sores, jaw stiffness, skin changes, lymphedema, tooth decay, and dysphagia from treatment effects on the muscles of mastication and swallowing. Radiation doses below 60 Gy are associated with improved functional swallowing outcomes (21), whereas each $10 \mathrm{~Gy}$ delivered above 55 Gy results in increasing rates of dysphagia (22). HPV related tumors are believed to be more sensitive to RT than HPV- tumors. In vitro and in vivo studies have demonstrated an intrinsic radio-sensitivity of HPV related head and neck cancers (23-25). The mechanism of this is not well understood. HPV-positive cancers may have a higher cellular radio-sensitivity than their HPV- counterparts due to cell cycle dysregulation and impaired DNA repair (26). Therefore, patients with HPV related disease may benefit from lower doses of RT while maintaining the same oncologic result. A number of trials have been conducted along this line of investigation.

In a prospective, multi-institutional phase II study published in 2015, Chera et al. reported on 43 patients treated with reduced dose radiation (60 Gy) along with weekly low-dose cisplatin. Patients were included if they had T0 to T3 and/or N0 to N2c HPV related disease and had a minimal smoking history. Following completion of treatment, patients were evaluated for complete clinical response based on physical and radiologic examination, and pathological clinical response based on planned surgical intervention. Patients underwent directed biopsies at the primary site and those with nodal disease underwent posttreatment neck dissection. The complete pathological response rate was $86 \%$, with decreased incidence of severe and very severe treatment toxicities (27). Long-term follow up from the study was published in 2018. The 3-year local control, regional control, cause-specific survival, distant metastasis-free survival, and overall survival rates were $100 \%, 100 \%, 100 \%, 100 \%$, and $95 \%$, respectively, with favorable quality of life outcomes (28).

A phase two clinical trial (NCT03323463) evaluating reduced dose RT is ongoing at Memorial Sloan Kettering Cancer Center. This is a prospective non-inferiority trial of major radiation dose de-escalation in patients with HPV+ T1-2/N1-2b OPSCC undergoing concurrent chemoradiation. Trial participants will receive $30 \mathrm{~Gy}$ radiation over three weeks with two cycles of standard chemotherapy, and will be compared to patients received a standard CRT regimen. Primary endpoint will be effectiveness of treatment at 2 years (5).

An ongoing trial (NCT03952585) combines both reduction in RT dosing and replacement of cisplatin with immune-checkpoint inhibitors. A randomized phase II/III trial is currently underway examining whether a reduced dose of RT and nivolumab works as well as standard dose RT and cisplatin in treating patients with HPV+ oropharyngeal cancer. Patients with AJCC $8^{\text {th }}$ edition T12, N1, M0 or T3, N0-N1, M0 disease are randomized to receive standard CRT, reduced dose RT with cisplatin, or reduced dose RT with nivolumab in place of cisplatin. Primary outcome measures will be progression-free survival at 2 years and quality of life measures (7).

Another line of investigation is to omit systemic therapy altogether for patients with locally advanced disease and treat patients with clinical low-risk disease with radiation alone. The NRG HN002 trial (NCT02254278) is ongoing and is randomizing non-smoking patients with p16-positive locally advanced OPSCC to receive 60 Gy RT with or without weekly cisplatin. The trial recently completed recruiting patients. The primary endpoint will be two-year progression free survival (7).

Given that patients with HPV-related OPSCC are, on average, younger and healthier than their HPV-negative counterparts, and that the side effects of radiation are long lasting, the focus on reduction of radiation volume and omission of toxic adjuvant therapy is appealing to maximize quality of life. These patients likely will have a long lifeexpectancy after surviving from their cancer. Therefore, this is an exciting line of de-escalation that has the likelihood to make a big impact on patient quality of life as HPV-related OPSCC cancer patients become long term survivors. 


\section{Induction chemotherapy followed by reduced radiation or chemoradiation}

Various trials have examined induction cisplatin-based chemotherapy followed by reduced radiation doses or volumes. Induction chemotherapy can be used for risk stratification with the assumption that adjuvant RT can be reduced in good responders.

In 2017, Marur et al. published the results of the ECOG 1308 trial which randomized 80 patients with stage III and IV (AJCC $7^{\text {th }}$ edition) disease to receive an induction regimen followed by either low (54 Gy) or standard dose IMRT with cetuximab, depending on the response to induction chemotherapy (29). Two-year progression free survival and overall survival were $80 \%$ and $94 \%$, respectively, for patients with $\mathrm{T} 3$ and/or $\mathrm{N} 2 \mathrm{~b}$ or below disease and those with a smoking history less than 10 years. At one year, significantly fewer patients who were treated with the reduced radiation dose reported difficulty swallowing ( $40 \%$ versus $89 \%, \mathrm{P}=0.011$ ) or impaired nutrition ( $10 \%$ versus $44 \%, \mathrm{P}=0.025)$.

In a single arm phase II clinical trial, Chen at al. reported on 44 patients with stage III and IV HPV related disease (AJCC $7^{\text {th }}$ edition) who were randomized to receive low (54 Gy in 27 fractions) or standard dose (60 Gy in 30 fractions) IMRT with paclitaxel, depending on response to an induction chemotherapy regimen (30). Progression free survival was $92 \%$ (95\% CI, 77-92).

The OPTIMA trial was a phase II radiation dose and volume de-escalation trial of 62 patients with HPV related OPSCC. After a trial of induction chemotherapy, low risk patients (those with $\leq \mathrm{T} 3, \leq \mathrm{N} 2 \mathrm{~B}$, and $\leq 10$ pack-year history) with $\geq 50 \%$ response received 50 Gy RT (RT50), while low risk patients with $30 \%-50 \%$ response or high-risk patients with $\geq 50 \%$ response received 45 Gy CRT (CRT45). Those with lesser responses received a standard chemoradiation regimen (CRT75). Two-year progression free survival and overall survival were $95 \%$ and $100 \%$ for low-risk patients and $94 \%$ and $97 \%$ for high-risk patients, respectively. Grade $3+$ mucositis occurred in 30\%, $63 \%$, and $91 \%$ of the RT50, CRT45, and CRT75 groups, respectively $(\mathrm{P}=0.004)$, while G-tube rates were $0 \%, 31 \%$, and $82 \%$ for RT50, CRT45, and CRT75 groups, respectively $(\mathrm{P}<0.0001)$ (31).

For standard treatment, induction remains highly selective for patients with bulky disease or to try to reduce the radiation field and therefore reduce radiation-associated morbidity. Induction chemotherapy also has to be given with caution so patients do not get too sick from their initial treatment and therefore are unable to continue on to their next step of therapy.

\section{Surgical approaches with de-escalated adjuvant therapy}

Traditional open surgical approaches to the oropharynx are associated with substantial morbidity as a result of poor access to the tumors. Advances in minimally invasive surgical therapy including transoral robotic surgery (TORS) and transoral laser microsurgery (TLM) have allowed for improved quality of life and functional outcomes with comparable oncologic results (32-35). For tumors amenable to minimally invasive surgical approaches, the goal is to spare or diminish the need for adjuvant treatment. Several prospective randomized surgical trials with de-escalated adjuvant therapy are underway.

MC1273 was a single-arm phase II trial evaluating reduced-dose of adjuvant RT following transoral surgical resection. Patients with HPV+ OPSCC with a less than 10 -pack year smoking history who underwent surgical resection with negative margins were included in the study. Intermediate risk patients received 30 Gy of adjuvant RT over 2 weeks, while patients with extranodal extension on final pathology received $36 \mathrm{~Gy}$. The 2-year locoregional tumor control rate was $96.2 \%$, with progression-free survival of $91.1 \%$ and overall survival of $98.7 \%$. Rates of grade 3 or worse toxicity at pre-RT and 1- and 2-year postRT were $2.5 \%, 0 \%$, and $0 \%$. Swallowing function improved slightly between pre-RT and 12 months post-RT (36).

ECOG 3311 (NCT01898494) is a phase II clinical trial enrolling patients to investigate reduced doses of adjuvant RT following transoral resection of intermediate-risk HPV+ tumors. Patients with unilateral, low stage (T1-T2) OPSCC and N1-N2b neck disease undergo transoral resection and neck dissection, followed by risk-based adjuvant treatment. Intermediate risk patients, defined as those with $\mathrm{N} 2$ or above disease with extra-nodal extension (ENE) $\leq 1 \mathrm{~mm}$ are randomized to receive reduced dose (50 Gy) RT or standard dose (60 Gy). Low stage patients receive no adjuvant treatment. Patients with evidence of ENE pre-operatively or with primary tumors that are likely to result in positive margins during transoral resection are excluded from the study (11).

The Post-Operative Adjuvant Treatment of HPVPositive Tumors (PATHOS) (NCT02215265) is another phase II trial examining the impact of transoral laser resection of tumors followed by reduced dose adjuvant 
RT on functional outcomes and survival. Patients undergo TLM resection of tumors, and are then randomized to reduced dose RT or standard dose RT (9).

The Adjuvant De-escalation, Extracapsular Spread, p16 Positive, Transoral (ADEPT) trial (NCT01687413) is a third phase II trial investigating reduced dose adjuvant RT and removal of chemotherapy from the adjuvant regimen of patients with extracapsular spread (ECS) on final pathology. All patients undergo transoral surgical resection and neck dissection. Patients are included in the trial if they have negative margins at the surgical site and must have ECS in nodal metastases on final pathology. Radiation volume is also not delivered to the primary bed in patients with completely resected $\mathrm{T} 1$ or $\mathrm{T} 2$ disease (13).

The DART-HPV (De-Escalated Adjuvant Radiation Therapy for HPV Associated Oropharynx Cancer) study (NCT02908477) is currently enrolling patients at the Mayo Clinic randomizing patients with HPV+ OPSCC who have undergone transoral resection and meet criteria for adjuvant treatment to receive standard adjuvant CRT or docetaxel with reduced adjuvant RT (30 Gy delivered over two weeks). The primary endpoint is treatment toxicity (10).

A single institution study at the University of Pennsylvania (NCT02159703) evaluated omission of adjuvant RT to the primary site following TORS and selective neck dissection for advanced stage HPV+ disease. This trial enrolled patients with pathologic $\mathrm{T} 1$ or $\mathrm{T} 2$ stage disease resected with TORS with negative margins and pathologic nodal stage N2a, N2b or N2c. Patients received adjuvant RT to the neck alone, while the primary site was spared. Two-year locoregional control and toxicity rates will be examined. Results have not yet been reported (37).

Finally, the ORATOR trial, an open label, phase II trial in which patients with OPSCC were randomized to receive radiotherapy or TORS with neck dissection recently published results. In this study, 68 adult patients with T1T2, N0-2 $(\leq 4 \mathrm{~cm})$ OPSCC were randomized to one of the two groups. The primary endpoint was swallowingrelated quality of life at one year as established using the MD Anderson Dysphagia Inventory (MDADI) score. They found that patients who underwent radiotherapy had a statistically significant improvement in swallowing one year after completing treatment compared to the surgery group (MDADI score 86.9 versus $80.1, \mathrm{P}=0.046$ ) (38). This suggests that radiotherapy may offer improved swallowing outcomes compared to surgery; however, further studies are needed to better understand if this is a clinically significant finding. The conclusion of this trial was particularly surprising given that previous studies suggested that surgery was more favorable compared to radiation in terms of swallowing outcomes. These studies were largely retrospective studies without formal toxicity evaluations. However, critics of the ORATOR trial note that the MDADI is only a subjective measurement of swallowing function and that more objective measures such as a videofluoroscopic swallow study should be used to evaluate swallowing function. As such, patients should be informed of both surgery and chemoradiation as options for their disease, and the benefits and risks of each should be weighed carefully by each patient and provider (39).

As these trials show, the role of surgery in treating HPVrelated OPSCC is constantly evolving. Traditional surgical paradigms are being challenged by the behavior of HPVrelated cancers. Further studies need to examine the impact of various pathologic factors in surgical outcomes including positive margins and extranodal extension. Because of the strong influence of HPV on biologic behavior and outcome, treatment algorithms must change and evolve, which is evidenced by the numerous clinical trials in this space.

\section{Conclusions}

Patients with HPV+ OPSCC fortunately have a good prognosis. Given the severe toxicities associated with standard, traditional treatment approaches to oropharyngeal cancer, there is benefit to investigating de-escalated therapies without compromising survival. Early results of trials comparing cetuximab to cisplatin showing no survival benefit published this year may temper enthusiasm for deescalation. However, results from trials of reduced doses of RT during concurrent CRT and induction chemotherapy followed by reduced RT based on induction response have demonstrated excellent oncologic and quality of life outcomes. Advances in minimally invasive surgery followed by reduced adjuvant regimens present exciting lines of investigation in ongoing clinical trials, which utilize advances in surgical technique to optimize quality of life and survival. As there is currently a lack of consistent randomized phase III clinical trial results, treatment deintensification is not recommended outside of ongoing clinical trials. It is important that de-intensification strategies are studied rigorously in order to best define clinical parameters to guide treatment choices. Any decrease in survival should not be tolerated; however, quality of life for patients with HPV+ OPSCC should also be emphasized. Further studies should also evaluate how patients are 
diagnosed with HPV-related oropharyngeal cancer, and if p16-staining is enough to stratify risk and prognosis. We will eagerly await the results of the numerous ongoing clinical trials in order to best help this patient population.

\section{Acknowledgments}

Funding: None.

\section{Footnote}

Reporting Checklist: The authors have completed the Narrative Review reporting checklist. Available at http:// dx.doi.org/10.21037/atm-20-2984

Conflicts of Interest: All authors have completed the ICMJE uniform disclosure form (available at http://dx.doi. org/10.21037/atm-20-2984). The authors have no conflicts of interest to declare.

Ethical Statement: The authors are accountable for all aspects of the work in ensuring that questions related to the accuracy or integrity of any part of the work are appropriately investigated and resolved.

Open Access Statement: This is an Open Access article distributed in accordance with the Creative Commons Attribution-NonCommercial-NoDerivs 4.0 International License (CC BY-NC-ND 4.0), which permits the noncommercial replication and distribution of the article with the strict proviso that no changes or edits are made and the original work is properly cited (including links to both the formal publication through the relevant DOI and the license). See: https://creativecommons.org/licenses/by-nc-nd/4.0/.

\section{References}

1. Gillison ML, Chaturvedi AK, Anderson WF, et al. Epidemiology of Human Papillomavirus-Positive Head and Neck Squamous Cell Carcinoma. J Clin Oncol 2015;33:3235-42.

2. Fakhry C, Westra WH, Li S, et al. Improved survival of patients with human papillomavirus-positive head and neck squamous cell carcinoma in a prospective clinical trial. J Natl Cancer Inst 2008;100:261-9.

3. Reduced-intensity Therapy for Oropharyngeal Cancer in Non-smoking HPV-16 Positive Patients. Secondary Reduced-intensity Therapy for Oropharyngeal Cancer in
Non-smoking HPV-16 Positive Patients. Available online: https://clinicaltrials.gov/ct2/show/NCT01663259

4. Ipilimumab, Nivolumab, and Radiation Therapy in Treating Patients With HPV Positive Advanced Oropharyngeal Squamous Cell Carcinoma. Secondary Ipilimumab, Nivolumab, and Radiation Therapy in Treating Patients With HPV Positive Advanced Oropharyngeal Squamous Cell Carcinoma. Available online: https://clinicaltrials.gov/ct2/show/NCT03799445

5. Major Radiation Reduction for HPV+ Oropharyngeal Carcinoma. Secondary Major Radiation Reduction for HPV+ Oropharyngeal Carcinoma. Available online: https://clinicaltrials.gov/ct2/show/NCT03323463

6. Reduced-Dose Intensity-Modulated Radiation Therapy With or Without Cisplatin in Treating Patients With Advanced Oropharyngeal Cancer. Secondary ReducedDose Intensity-Modulated Radiation Therapy With or Without Cisplatin in Treating Patients With Advanced Oropharyngeal Cancer. Available online: https:// clinicaltrials.gov/ct2/show/NCT02254278

7. De-intensified Radiation Therapy With Chemotherapy (Cisplatin) or Immunotherapy (Nivolumab) in Treating Patients With Early-Stage, HPV-Positive, Non-Smoking Associated Oropharyngeal Cancer. Secondary Deintensified Radiation Therapy With Chemotherapy (Cisplatin) or Immunotherapy (Nivolumab) in Treating Patients With Early-Stage, HPV-Positive, Non-Smoking Associated Oropharyngeal Cancer. Available online: https://clinicaltrials.gov/ct2/show/NCT03952585

8. De-escalation Protocols in HPV-related Oropharyngeal Carcinoma in Chinese Populations. Secondary Deescalation Protocols in HPV-related Oropharyngeal Carcinoma in Chinese Populations. Available online: https://clinicaltrials.gov/ct2/show/NCT04012502

9. Post-operative Adjuvant Treatment for HPV-positive Tumours (PATHOS). Secondary Post-operative Adjuvant Treatment for HPV-positive Tumours (PATHOS). Available online: https://clinicaltrials.gov/ct2/show/ NCT02215265

10. Evaluation of De-escalated Adjuvant Radiation Therapy for Human Papillomavirus (HPV)-Associated Oropharynx Cancer. Secondary Evaluation of De-escalated Adjuvant Radiation Therapy for Human Papillomavirus (HPV)Associated Oropharynx Cancer. Available online: https:// clinicaltrials.gov/ct2/show/NCT02908477

11. Transoral Surgery Followed By Low-Dose or StandardDose Radiation Therapy With or Without Chemotherapy in Treating Patients With HPV Positive Stage III-IVA 
Oropharyngeal Cancer. Secondary Transoral Surgery Followed By Low-Dose or Standard-Dose Radiation Therapy With or Without Chemotherapy in Treating Patients With HPV Positive Stage III-IVA Oropharyngeal Cancer. Available online: https://clinicaltrials.gov/ct2/ show/NCT01898494

12. TORS De-Intensification Protocol Version 2.0: Dose and Volume Reduction in the Neck. Secondary TORS De-Intensification Protocol Version 2.0: Dose and Volume Reduction in the Neck. Available online: https:// clinicaltrials.gov/ct2/show/NCT03729518

13. Post Operative Adjuvant Therapy De-intensification Trial for Human Papillomavirus-related, p16+ Oropharynx Cancer (ADEPT). Secondary Post Operative Adjuvant Therapy De-intensification Trial for Human Papillomavirus-related, p16+ Oropharynx Cancer (ADEPT). Available online: https://clinicaltrials.gov/ct2/ show/NCT01687413

14. De-escalation of Adjuvant Radio (Chemo) Therapy for HPV-positive Head-neck Squamous Cell Carcinomas (DELPHI). Secondary De-escalation of Adjuvant Radio (Chemo) Therapy for HPV-positive Head-neck Squamous Cell Carcinomas (DELPHI). Available online: https:// clinicaltrials.gov/ct2/show/NCT03396718

15. Bonner JA, Harari PM, Giralt J, et al. Radiotherapy plus cetuximab for squamous-cell carcinoma of the head and neck. N Engl J Med 2006;354:567-78.

16. Bonner JA, Harari PM, Giralt J, et al. Radiotherapy plus cetuximab for locoregionally advanced head and neck cancer: 5-year survival data from a phase 3 randomised trial, and relation between cetuximab-induced rash and survival. Lancet Oncol 2010;11:21-8.

17. Cancer Genome Atlas Network. Comprehensive genomic characterization of head and neck squamous cell carcinomas. Nature 2015;517:576-82.

18. Gillison ML, Trotti AM, Harris J, et al. Radiotherapy plus cetuximab or cisplatin in human papillomavirus-positive oropharyngeal cancer (NRG Oncology RTOG 1016): a randomised, multicentre, non-inferiority trial. Lancet 2019;393:40-50.

19. Mehanna H, Robinson M, Hartley A, et al. Radiotherapy plus cisplatin or cetuximab in low-risk human papillomavirus-positive oropharyngeal cancer (DeESCALaTE HPV): an open-label randomised controlled phase 3 trial. Lancet 2019;393:51-60.

20. Hong AM, Ferguson P, Dodds T, et al. Significant association of PD-L1 expression with human papillomavirus positivity and its prognostic impact in oropharyngeal cancer. Oral Oncol 2019;92:33-9.

21. Mortensen HR, Jensen K, Aksglaede K, et al. Late dysphagia after IMRT for head and neck cancer and correlation with dose-volume parameters. Radiother Oncol 2013;107:288-94.

22. Levendag PC, Teguh DN, Voet P, et al. Dysphagia disorders in patients with cancer of the oropharynx are significantly affected by the radiation therapy dose to the superior and middle constrictor muscle: a dose-effect relationship. Radiother Oncol 2007;85:64-73.

23. Kimple RJ, Smith MA, Blitzer GC, et al. Enhanced radiation sensitivity in $\mathrm{HPV}$-positive head and neck cancer. Cancer Res 2013;73:4791-800.

24. Swick AD, Chatterjee A, De Costa AM, et al. Modulation of therapeutic sensitivity by human papillomavirus. Radiother Oncol 2015;116:342-5.

25. Park JW, Nickel KP, Torres AD, et al. Human papillomavirus type $16 \mathrm{E} 7$ oncoprotein causes a delay in repair of DNA damage. Radiother Oncol 2014;113:337-44.

26. Arenz A, Ziemann F, Mayer C, et al. Increased radiosensitivity of $\mathrm{HPV}$-positive head and neck cancer cell lines due to cell cycle dysregulation and induction of apoptosis. Strahlenther Onkol 2014;190:839-46.

27. Chera BS, Amdur RJ, Tepper J, et al. Phase 2 Trial of De-intensified Chemoradiation Therapy for FavorableRisk Human Papillomavirus-Associated Oropharyngeal Squamous Cell Carcinoma. Int J Radiat Oncol Biol Phys 2015;93:976-85.

28. Chera BS, Amdur RJ, Tepper JE, et al. Mature results of a prospective study of deintensified chemoradiotherapy for low-risk human papillomavirus-associated oropharyngeal squamous cell carcinoma. Cancer 2018;124:2347-54.

29. Marur S, Li S, Cmelak AJ, et al. E1308: Phase II Trial of Induction Chemotherapy Followed by Reduced-Dose Radiation and Weekly Cetuximab in Patients With HPVAssociated Resectable Squamous Cell Carcinoma of the Oropharynx- ECOG-ACRIN Cancer Research Group. J Clin Oncol 2017;35:490-7.

30. Chen AM, Felix C, Wang PC, et al. Reduced-dose radiotherapy for human papillomavirus-associated squamous-cell carcinoma of the oropharynx: a single-arm, phase 2 study. Lancet Oncol 2017;18:803-11.

31. Seiwert TY, Foster CC, Blair EA, et al. OPTIMA: a phase II dose and volume de-escalation trial for human papillomavirus-positive oropharyngeal cancer. Ann Oncol 2019;30:297-302.

32. Moore EJ, Olsen SM, Laborde RR, et al. Long-term functional and oncologic results of transoral robotic 
surgery for oropharyngeal squamous cell carcinoma. Mayo Clin Proc 2012;87:219-25.

33. Genden EM, Kotz T, Tong CC, et al. Transoral robotic resection and reconstruction for head and neck cancer. Laryngoscope 2011;121:1668-74.

34. Weinstein GS, O'Malley BW Jr, Magnuson JS, et al. Transoral robotic surgery: a multicenter study to assess feasibility, safety, and surgical margins. Laryngoscope 2012;122:1701-7.

35. Dowthwaite SA, Franklin JH, Palma DA, et al. The role of transoral robotic surgery in the management of oropharyngeal cancer: a review of the literature. ISRN Oncol 2012;2012:945162.

36. Ma DJ, Price KA, Moore EJ, et al. Phase II Evaluation of Aggressive Dose De-Escalation for Adjuvant Chemoradiotherapy in Human Papillomavirus-Associated Oropharynx Squamous Cell Carcinoma. J Clin Oncol 2019;37:1909-18.

37. A Single-arm Phase II Study of Post-Transoral Robotic Surgery (TORS) Alone to the Primary Tumor Site and

Cite this article as: Strohl MP, Wai KC, Ha PK. Deintensification strategies in HPV-related oropharyngeal squamous cell carcinoma-a narrative review. Ann Transl Med 2020;8(23):1601. doi: 10.21037/atm-20-2984
Selective Neck Dissection (SND) Followed by Adjuvant Radiation Therapy (+/- Chemotherapy) to the Regional Nodes for Advanced Stage, Human Papilloma Virus (HPV) Positive, Oropharyngeal Cancer. Secondary A Singlearm Phase II Study of Post-Transoral Robotic Surgery (TORS) Alone to the Primary Tumor Site and Selective Neck Dissection (SND) Followed by Adjuvant Radiation Therapy (+/- Chemotherapy) to the Regional Nodes for Advanced Stage, Human Papilloma Virus (HPV) Positive, Oropharyngeal Cancer. Available online: https:// clinicaltrials.gov/ct2/show/NCT02159703

38. Nichols AC, Theurer J, Prisman E, et al. Radiotherapy versus transoral robotic surgery and neck dissection for oropharyngeal squamous cell carcinoma (ORATOR): an open-label, phase 2, randomised trial. Lancet Oncol 2019;20:1349-59.

39. Grégoire V, Nicolai P. Choosing surgery or radiotherapy for oropharyngeal squamous cell carcinoma: is the issue definitely settled? Lancet Oncol 2019;20:1328-9. 